\title{
Existence results for some nonlinear integral equations
}

\author{
Monica Lauran
}




\title{
EXISTENCE RESULTS FOR SOME NONLINEAR INTEGRAL EQUATIONS
}

\author{
MONICA LAURAN \\ Received 30 June, 2011
}

\begin{abstract}
In this paper we shall establish sufficient conditions for the existence of solutions of the integral equation of Volterra type and for its solvability in Banach space and $C_{L}$. The main tools used in our study are the nonexpansive operator technique, contraction principle and Schaefer's fixed point theorem.
\end{abstract}

2000 Mathematics Subject Classification: 45B05; 45D05; 47H10

Keywords: nonlinear integral equation, existence of solutions, nonexpansiv mapping

\section{INTRODUCTION}

The theory of integral equations has many applications in describing numerous events and problems of the real world. For example, integral equations are often applicable in engineering, mathematical physics, economics and biology. It is a well known fact that the nonlinear quadric equations are often encountered in various applications.

Fixed point theorems used in nonlinear functional analysis allows us, in general, to obtain existence theorems concerning the investigated functional-operator equations. In this paper we study the existence of solutions of nonlinear integral equation of Volterra type by using the concepts of nonexpansive operators, contraction principles and the Schaefer's fixed point theorem. The result generalize previous results of $[1-3,6,7]$.

\section{PRELIMinaries}

In this section, we introduce notations, definitions and preliminary facts which are used throughout this paper. By $C(J, R)$ we denote the Banach space of all continuous functions from $J$ to $\mathbb{R}$ with norm

$$
\|y\|:=\sup \{|y(t)|: t \in J\}
$$

We can now formulate one of the most important fixed point theorems that will used throughout the paper. These theorems can be found in papers such as $[3,5,8]$. 
Definition 1 ([3]). Let $(X, d)$ be a metric space and $B \subseteq C(X, \mathbb{R})$. $B$ is a equicontinuous means if for all $\varepsilon>0$ there exists $\delta>0$ such that for all $f \in B, x, a \in X$,

$$
d(x, a)<\delta \Rightarrow|f(x)-f(a)|<\varepsilon .
$$

Theorem 1 (Arzelá-Ascoli, [5]). Let $(X, d)$ be a compact metric space and let $B \subseteq C(X, \mathbb{R})$. Then $B$ is compact if and only if $B$ is closed, bounded and equicontinuous.

Theorem 2 (Schaefer, [5]). Let $X$ be a normed linear space. If $A: X \rightarrow X$ is a completly continuous map, then either the subset

$$
\{x \in X: x=\lambda A x \text {, for some } 0 \leq \lambda \leq 1\}
$$

is bounded or A has a fixed point.

Theorem 3 (Schaefer, [5]). Let $X$ be a Banach space and $K \subset X$ a nonempty, convex, compact subset of $X$. If $T: K \rightarrow K$ is a continuous operator, then $T$ has at least one fixed point in $K$.

\section{MAIN RESULTS}

Consider the following nonlinear functional-integral equation

$$
x(t)=g(t, x(t))+h(t, x(t)) \int_{0}^{T} f(t, s, x(s)) d s, T \geq 0 .
$$

Let $J=[0, T]$ and

$$
\begin{gathered}
C_{L}=\left\{x \in C(J, J)|| x\left(t_{1}\right)-x\left(t_{2}\right)|\leq L \cdot| t_{1}-t_{2} \mid,\right. \\
\left.(\forall) t_{1}, t_{2} \in \mathbb{R}_{+}\right\}, L>0
\end{gathered}
$$

Our first result is based on the Banach fixed point theorem.

Theorem 4. Assume that

(H) $f: \mathbb{R}_{+} \times \mathbb{R}_{+} \times \mathbb{R} \rightarrow \mathbb{R} \quad g, h: \mathbb{R}_{+} \times \mathbb{R} \rightarrow \mathbb{R}$ are given continuous functions. $\left(H_{2}\right)$ There exists a constant $L_{1}>0$ such that

$$
|g(t, x)-g(t, y)| \leq L_{1} \cdot|x-y|, \quad(\forall) t \in J, x, y \in \mathbb{R} .
$$

$\left(H_{3}\right)$ There exists a constant $L_{2}>0$ such that

$$
|h(t, x)-h(t, y)| \leq L_{2} \cdot|x-y|, \quad(\forall) t \in J, x, y \in \mathbb{R} .
$$

$\left(H_{4}\right)$ Then exists a constant $L_{3}>0$ such that

$$
|f(t, s, x)-f(t, s, y)| \leq L_{3} \cdot|x-y|, \quad(\forall) t, s \in J, x, y \in \mathbb{R} .
$$




$$
\begin{aligned}
& \text { If } \\
& \sup _{t \geq 0}\left\{L_{1}+L_{2} \cdot \int_{0}^{T}|f(t, s, x(s))| d s+L_{3} \cdot T \cdot|h(t, x(t))|\right\} \leq k<1,(\forall) t \in J(*)
\end{aligned}
$$

then equation 3.1 has a unique solution on $J=[0, T]$.

Proof. Transform the equation 3.1 into a fixed point problem.

Consider the operator

$$
F: C(J, \mathbb{R}) \rightarrow C(J, \mathbb{R})
$$

defined by

$$
F(x)(t)=g(t, x(t))+h(t, x(t)) \int_{0}^{T} f(t, s, x(s)) d s .
$$

The fixed point of the operator $F$ are solution of equation 3.1. We shall use the Banach contraction principle to prove that $F$ has a fixed point.

Let $x, y \in C(J, \mathbb{R})$. We have

$$
\begin{gathered}
|(F x)(t)-(F y)(t)| \leq|g(t, x(t))-g(t, y(t))|+ \\
\mid h(t, x(t)) \int_{0}^{T} f(t, s, x(s)) d s-h(t, y(t)) \int_{0}^{T} f(t, s, y(s)) d s+ \\
+h(t, x(t)) \int_{0}^{T} f(t, s, y(s)) d s-h(t, x(t)) \int_{0}^{T} f(t, s, y(s)) d s \mid \leq \\
\leq L_{1}|x(t)-y(t)|+|h(t, x(t))| \cdot \int_{0}^{T}|f(t, s, x(s)) d s-f(t, s, y(s))| d s+ \\
+|h(t, x(t))-h(t, y(t))| \cdot \int_{0}^{T} f(t, s, y(s)) d s \leq L_{1}|x(t)-y(t)|+ \\
+L_{3} \cdot|h(t, x(t))| \cdot \int_{0}^{t}|x(s)-y(s)| d s+L_{2} \cdot|x(t)-y(t)| \cdot \int_{0}^{T}|f(t, s, y(s))| d s .
\end{gathered}
$$

Thus

$$
\|F x-F y\| \leq\left(L_{1}+L_{2} \cdot \int_{0}^{T}|f(t, s, y(s))| d s+L_{3} \cdot T \cdot|h(t, x(t))|\right) \cdot\|x-y\| .
$$


Consequently $F$ is a contraction. As a consequence of the Banach fixed point theorem, we deduce that $F$ has a fixed point which is a solution of equation 3.1.

The second result is based on Schaefer's fixed point theorem.

Theorem 5. Assume the following conditions in equation 3.1:

$\left(H_{1}\right)$. The functions

$$
\begin{aligned}
& g: \mathbb{R}_{+} \times \mathbb{R} \rightarrow \mathbb{R} \\
& h: \mathbb{R}_{+} \times \mathbb{R} \rightarrow \mathbb{R} \\
& f: \mathbb{R}_{+} \times \mathbb{R}_{+} \times \mathbb{R} \rightarrow \mathbb{R}
\end{aligned}
$$

are continuous.

$\left(\mathrm{H}_{2}\right)$. If $\mathrm{L}$ is the Lipschitz constant involved in 3.2, then

$$
\left|\int_{0}^{T} f\left(t_{1}, s, x(s)\right) d s-\int_{0}^{T} f\left(t_{2}, s, x(s)\right) d s\right| \leq L \cdot\left|t_{1}-t_{2}\right|,
$$

for each $(\forall) t_{1}, t_{2}, s \in J$ and $x \in \mathbb{R}$ and

$$
Q=\max _{t \in J}\left\{\left|h(t, x(t)) \int_{0}^{T} f(t, s, x(s)) d s\right|\right\},(\forall) t, s \in J \text { and } x \in \mathbb{R} .
$$

$\left(H_{3}\right)$. There exists the constants $m, M, p \in \mathbb{R}$ such that

$$
\begin{gathered}
|g(t, x)-g(t, y)| \leq m \cdot|x-y| \text { for each } x, y \in \mathbb{R} \text { and } t \in J, \\
|g(t, x)-g(s, x)| \leq M \cdot L \cdot|t-s| \text { and }(\forall) t, s \in J, x \in \mathbb{R}, \\
|g(t, x(t))| \leq p,(\forall) t, s \in J, x \in \mathbb{R} .
\end{gathered}
$$

$\left(H_{4}\right)$. There exists the constants $n, N \in \mathbb{R}$ such that

$$
\begin{aligned}
& |h(t, x)-h(t, y)| \leq n \cdot|x-y|(\forall) x, y \in \mathbb{R} \text { and } t \in J, \\
& |h(t, x)-h(s, x)| \leq N \cdot L \cdot|t-s|(\forall) t, s \in J \text { and } x \in \mathbb{R}
\end{aligned}
$$

$\left(H_{5}\right)$. There exist the constant $k \in(0,1]$ such that

$$
\sup _{t \in J}\left\{m+M+(n+N) \int_{0}^{T}|f(t,, s, x(t))| d s+|h(t, x(t))|\right\} \leq k .
$$

Then the equation 3.1 has at least one solution on $C_{L}$.

Proof. It is known, (see Lemma 1 in [4]), that $C_{L}$ is nonempty and convex, moreover, it is a compact subset of the Banach space $(C[a, b],\|\cdot\|)$, where $\|\cdot\|$ is the usual supremum norm.

Now we transform equation 3.1 into a fixed point problem. 
Consider the integral operator

$$
\begin{gathered}
F: C_{L} \rightarrow C(J, \mathbb{R}) \\
(F x)(t)=g(t, x(t))+h(t, x(t)) \int_{0}^{T} f(t, s, x(s)) d s .
\end{gathered}
$$

Clearly, the fixed points of the operator $F$ are solutions of equation 3.1. We shall use Schaefer's fixed point theorem to prove that $F$ has a fixed point.

Step 1. $F$ is continuous

Let $\left\{x_{n}\right\}$ be o sequence such that $x_{n} \rightarrow x$ in $C(J, \mathbb{R})$. Then for each $t \in J$

$$
\begin{gathered}
\left|F\left(x_{n}\right)(t)-F(x)(t)\right| \\
\leq\left|g\left(t, x_{n}(t)\right)-g(t, x(t))\right|+\left|h\left(t, x_{n}(t)\right)\right| \cdot \int_{0}^{T} \mid f\left(t, s, x_{n}(s)\right)-f(t, s, x(s)) d s+ \\
+\left|h\left(t, x_{n}(t)\right)-h(t, x(t))\right| \cdot \int_{0}^{T} \mid f(t, s, x(s) \mid d s .
\end{gathered}
$$

Since $f, g$ and $h$ are continuous functions, we have

$$
\left\|F x_{n}-F x\right\| \rightarrow 0 \text {, as } n \rightarrow \infty .
$$

Step 2. $F$ maps bounded sets in $C_{L}$ into bounded sets in $C(J, \mathbb{R})$.

Indeed, it is enough to show for any $\eta>0$, there exists a positive constant $l$ such that for each $x \in B_{\eta}=\left\{x \in C_{L}:\|x\| \leq \eta\right\}$, we have $\|F x\| \leq l$.

By $\left(\mathrm{H}_{3}\right)-\left(\mathrm{H}_{4}\right)$ we have for each $t \in J$

$$
|(F x)(t)| \leq|g(t, x(t))|+\left|h(t, x(t)) \int_{0}^{T} f(t, s, x(s)) d s\right| \leq p+Q .
$$

Thus

$$
\|F x\| \leq p+Q:=l .
$$

Step 3. $F$ maps bounded sets into equicontinuous sets of $C_{L}$.

$$
\begin{gathered}
\left|(F x)\left(t_{1}\right)-(F x)\left(t_{2}\right)\right| \leq \mid g\left(t_{1}, x\left(t_{1}\right)-g\left(t_{2}, x\left(t_{2}\right)\right) \mid+\right. \\
+\left|h\left(t_{1}, x\left(t_{1}\right)\right) \int_{0}^{T} f\left(t_{1}, s, x(s)\right) d s-h\left(t_{2}, x\left(t_{2}\right)\right) \int_{0}^{T} f\left(t_{2}, s, x(s)\right) d s\right| \leq \\
\leq\left|g\left(t_{1}, x\left(t_{1}\right)\right)-g\left(t_{2}, x\left(t_{1}\right)\right)\right|+\left|g\left(t_{2}, x\left(t_{1}\right)\right)-g\left(t_{2}, x\left(t_{2}\right)\right)\right|+
\end{gathered}
$$




$$
\begin{aligned}
& +\left|h\left(t_{1}, x\left(t_{2}\right)\right)-h\left(t_{2}, x\left(t_{1}\right)\right)\right| \cdot \int_{0}^{T}\left|f\left(t_{1}, s, x(s)\right)\right| d s+ \\
& +\left|h\left(t_{2}, x\left(t_{1}\right)\right)\right| \cdot\left|\int_{0}^{T} f\left(t_{1}, s, x(s)\right) d s-\int_{0}^{T} f\left(t_{2}, s, x(s)\right) d s\right| \leq \\
& \leq M \cdot L \cdot\left|t_{1}-t_{2}\right|+m \cdot\left|x\left(t_{1}\right)-x\left(t_{2}\right)\right|+ \\
& +\mid h\left(t_{1}, x\left(t_{2}\right)-h\left(t_{1}, x\left(t_{1}\right)\left|\cdot \int_{0}^{T}\right| f\left(t_{1}, s, x(s)\right) \mid d s+\right.\right. \\
& +\left|h\left(t_{1}, x\left(t_{1}\right)\right)-h\left(t_{2}, x\left(t_{1}\right)\right)\right| \cdot \int_{0}^{T}\left|f\left(t_{1}, s, x(s)\right)\right| d s+ \\
& +\left|h\left(t_{2}, x\left(t_{1}\right)\right)\right| \cdot\left|\int_{0}^{T} f\left(t_{1}, s, x\right) d s-\int_{0}^{T} f\left(t_{2}, s, x\right) d s\right| \leq \\
& \leq M \cdot L \cdot\left|t_{1}-t_{2}\right|+m \cdot L \cdot\left|t_{1}-t_{2}\right|+ \\
& +(N \cdot L+n \cdot L) \cdot\left|t_{1}-t_{2}\right| \cdot \int_{0}^{T}\left|f\left(t_{1}, s, x\right)\right| d s+ \\
& +\left|h\left(t_{2}, x\left(t_{1}\right)\right)\right| \cdot L \cdot\left|t_{1}-t_{2}\right|= \\
& =L \cdot\left|t_{1}-t_{2}\right|\left[m+M+(n+N) \int_{0}^{T}\left|f\left(t_{1}, s, x\right)\right| d s+\left|h\left(t_{2}, x\left(t_{1}\right)\right)\right|\right] \leq \\
& \leq L \cdot\left|t_{1}-t_{2}\right|
\end{aligned}
$$

So $F x \in C_{L},(\forall) x \in C_{L}$. Therefore $T: C_{L} \rightarrow C_{L}$.

As $t_{1} \rightarrow t_{2}$, the right-hand side of the above inequality tends to zero. As a consequence of steps 1 ot 3 , by using the Arzelá-Ascoli theorem, we can conclude that $F: C_{L} \rightarrow C_{L}$ is continuous and completely continuous.

Step 4. A priori bounds.

Now it remains to show that the set

$$
\varepsilon=\left\{x \in C_{L}: x=\lambda F x \text { for some } 0 \leq \lambda \leq 1\right\}
$$

is bounded.

Let $x \in \varepsilon$ then $x=\lambda F x$ for some $0 \leq \lambda \leq 1$ 


$$
|x(t)| \leq|g(t, x(t))|+\left|h(t, x(t)) \int_{0}^{T} f(t, s, x(s)) d s\right| \leq p+Q:=R
$$

As a consequence of Schaefer's fixed point theorem we deduce that $F$ has a fixed point which is a solution of equation 3.1.

In the following theorem we shall give an existence result for equation 3.1 by means of Schauder's fixed point theorem.

Theorem 6. Assume that $\left(H_{1}\right),\left(H_{3}\right),\left(H_{4}\right)$ in theorem 5 hold and $\left(H_{2}^{\prime}\right)$ There exists a constant $L^{\prime}$ such that

$$
|f(t, s, u)-f(t, s, v)| \leq L^{\prime} \cdot|u-v|, \text { for each } t, s \in J, u, v \in \mathbb{R} .
$$

If

$$
\sup _{t \geq 0}\left\{m+n \int_{0}^{t} \mid f\left(t, s, x(s)\left|d s+t \cdot L^{\prime} \cdot\right| h(t, x(t)) \mid\right\} \leq 1\right.
$$

or

$$
\lim _{t \rightarrow \infty} t \cdot|h(t, x(t))|=0 \text { uniformly with respect to } x \in C_{L},
$$

then the equation 3.1 has at least one solution in $C_{L}$.

Proof. Using the proof of theorem 4 and theorem 5, one can prove that $F$ is nonexpansive. Applying the Schauder's fixed point theorem, we deduce that $F$ has at least one fixed point which is a solution of equation 3.1.

\section{AN EXAMPLE}

In this section we give an example to illustrate the usefulness of our main results. Let us consider the following nonlinear integral equation

$$
x(t)=\frac{1}{t^{2}+9} \cdot x(t)+\cos (t x(t)) \int_{0}^{T} \frac{e^{-t}}{1+e^{t}} \cdot \frac{|x(s)|}{1+|x(s)|} d s
$$

In this case we have

$$
\begin{gathered}
g: \mathbb{R}_{+} \rightarrow \mathbb{R}, g(t, x(t))=\frac{1}{t^{2}+9} \cdot x(t), \text { for all } t \in J, \\
h: \mathbb{R}_{+} \rightarrow \mathbb{R}, h(t, x(t))=\cos (t x(t)), \text { for all } t \in J,
\end{gathered}
$$

and

$$
f: \mathbb{R}_{+} \times \mathbb{R}_{+} \times \mathbb{R} \rightarrow \mathbb{R}, f(t, s, x(s))=\frac{e^{-t}}{1+e^{t}} \cdot \frac{|x(s)|}{1+|x(s)|}, \text { for all } t, s \in J .
$$


We are interested to study the solution $x(t)$ belonging to

$$
C_{1}=\left\{x \in C(J, J):\left|x\left(t_{1}\right)-x\left(t_{2}\right)\right| \leq\left|t_{1}-t_{2}\right|,(\forall) t_{1}, t_{2} \in J\right\},
$$

which means $L=1$. We have $L_{1}=\frac{1}{9}, L_{2}=\frac{1}{2}, L_{3}=\frac{1}{2}$ in Theorem 4 .

If $L_{1}<k<1$ in 4, the equation 4.1 has a unique solution in $J=[0, T]$.

For $T$, it is especially convenient to set $m=\frac{1}{9}, M=\frac{1}{36}, Q=\frac{1}{2}, n=\frac{1}{2}, N=\frac{1}{2}$ and $L^{\prime}=\frac{1}{2}$ in theorem 6 . Then equation 4.1 has at least one solution in $C_{1}$.

\section{REFERENCES}

[1] K. Balachandran and M. D. Julie, "Asymptotic stability of solutions of nonlinear integral equations," Nonlinear Funct. Anal. Appl., vol. 13, no. 2, pp. 311-322, 2008.

[2] J. Banas, J. Rocha, and K. B. Sadarangani, "Solvability of a nonlinear integral equation of Volterra type," J. Comput. Appl. Math., vol. 157, no. 1, pp. 31-48, 2003.

[3] V. Berinde, Iterative approximation of fixed points. 2nd revised and enlarged ed., ser. Lecture Notes in Mathematics. Berlin: Springer, 2007, vol. 1912.

[4] V. Berinde, "Existence and approximation of solutions of some first order iterative differential equations," Math. Notes, Miskolc, vol. 11, no. 1, pp. 13-26, 2010.

[5] A. Buică, "Existence and continuous dependence of solutions of some functional-differential equations," Babeş-Bolyai Univ., Fac. Math. Comput. Sci., Res. Semin., Prepr., vol. 1995, no. 3, pp. 1-13, 1995.

[6] A. Granas and J. Dugundji, Fixed point theory, ser. Springer Monographs in Mathematics. New York: Springer, 2003.

[7] M. Lauran, "Existence results for some integral equation with modified argument," General Mathematics, vol. 19, no. 3, pp. 85-92, 2011.

[8] D. O'Regan, "Existence results for nonlinear integral equations," J. Math. Anal. Appl., vol. 192, no. 3, pp. 705-726, 1995.

Author's address

Monica Lauran

North University of Baia Mare, Department of Mathematics and Computer Science, Victoriei 76, 430122 Baia Mare, Romania

E-mail address: lauranmonica@yahoo.com 